\title{
Does Intralesional Injection of Clostridium Histolyticum affect the Treatment Practice of Peyronie's Disease?
}

\author{
Alexander Greenstein, Boaz Appel, Valentin Shabataev, Ravit Yehieli-Cohen and Ilan Gruenwald* \\ Neurourology Unit, Rambam Health care Campus
}

*Corresponding author: Ilan Gruenwald, Department of Neuro-Urology, Rambam Health Care Campus, and Ruth and Bruce Rappaport Faculty of Medicine, Technion, Haifa, Israel.

Received Date: February 22, 2021

Published Date: March 23, 2021

\begin{abstract}
Introduction: Penile curvature and pain during erection present a significant bothersome issue for men with Peyronie's disease and their partners. Clostridium histolyticum was approved for intralesional-injection in patients with Peyronie's disease and penile curvature.

Aim: We aimed primarily to determine whether the introduction of intralesional-injection of clostridium histolyticum affects the treatment pattern of Peyronie's disease at a single academic medical center.

Material and methods: Medical records of patients evaluated for penile curvature related to Peyronie's disease were retrospectively reviewed. Patients were allocated into two groups: those who received consultation prior to intralesional-injection availability, and those who received consultation after intralesional-injection availability. Treatment choices were summarized as percentages of each group. Comparison between the groups regarding the proportion of patients who chose to undergo specific treatment was performed using a Chi-square test.

Results: Two hundred and twenty-seven patients were evaluated. Fifty-nine were evaluated before the introduction of intralesional-injection of clostridium histolyticum (group A) while 168 patients were evaluated post-introduction of intralesional-injection of clostridium histolyticum (group B). After the first discussion on management, 32 (54.2\%) group A and 105 (62.5\%) group B patients decided not to pursue treatment nor follow-up. No statistically significant difference in percentage of patients undergoing any treatment was observed between the groups ( $p>0.05$ ). Of the remaining 27 patients in group A, 10 patients (37.0\%) underwent surgery. Of the 63 remaining patients in group B, $14(22.2 \%)$ underwent surgery. No statistically significant difference in percentage of patients undergoing surgery was observed between the 2 groups. Of the remaining patients in group B, only $12(19.0 \%)$ were treated with intralesional-injection of clostridium histolyticum, and the rest, thirty-seven (59\%) patients, elected to continue follow-up without treatment.
\end{abstract}

Conclusion: The addition of intralesional-injection of clostridium histolyticum to Peyronie's disease treatment arsenal did not significantly alter the percentage of patients choosing to undergo surgery.

\section{Introduction}

Since the initial description of the condition by the François Gigot de la Peyronie in 1743, the etiology and the optimal treatment are still unidentified. PD is defined as fibrous plaques on the dorsolateral aspect of the penis, commonly involving the membrane (tunica albuginea) that surrounds the erectile tissue [1]. The fibrous plaque may eventually cause a painful deformity of the shaft or constriction of the urethra. The fibrous plaque is characterized histologically by the presence of spindle-shaped fibroblasts, causing the penis to bend during erection. [1]. It usually affects men aged 40-70 years and the majority (70\%) of them have dorsal curvature with a mean curvature angle of $42 \pm 22$ degrees [1]. Penile size and shape are of significant concern among men of all ages [2,3]. Penile deformity, painful erections and erectile dysfunction are the major causes of anxiety and frustration in men with Peyronie's disease (PD) [4]. Therefore, men with penile deformity related to PD often seek professional medical advice 
in search of the optimal potential treatment for this bothersome condition. Although surgery is considered the gold standard for the treatment of penile curvature related to $\mathrm{PD}$, some men are reluctant to undergo any surgical procedure of the penis and ultimately avoid definitive treatment. In 2013, the FDA approved clostridium histolyticum collagenase enzyme $(\mathrm{CCH})$ for intralesional injection (ILI), broadening the arsenal for the treatment of men with penile curvature related to PD. Our basic assumption was that, following the advent of ILIC, a substantial proportion of men with PD will prefer this mode of treatment mainly due to its obvious advantage as a conservative therapy. The primary aim of this study was to determine whether the introduction of ILI with CCH (ILIC) in 2016 in Israel affects the treatment pattern of PD, by comparing two groups of patients: one pre-CCH availability versus post-CCH availability at a single academic medical center.

\section{Material and Methods}

\section{Study design and population}

This study a retrospective cohort analysis of adult patients attending clinic at the Neuro-urology Unit, Rambam Healthcare Campus for PD. The study was approved by the Helsinki committee of the medical center, all adult men who underwent evaluation for stable PD from January 1st, 2013 to January 12th, 2019 were included in this study. Medical records were retrieved and reviewed directly by the study authors. To determine whether the availability of ILI CCH affected the proportion of patients choosing treatment and specifically surgery for PD. The cohort was divided into two groups based on ILI CCH availability and compared: Group A comprised of all PD consults prior to the introduction of ILI CCH (January 1st, 2013 to March 31st, 2016) and Group B comprised of all PD consults after introduction of ILI CCH (April 1st, 2016 to January 12th, 2019).

\section{Result}

\section{Clinical disease assessments}

Stable PD was defined as penile curvature without pain and no change in curvature's angle during erection during the past six months. Patients underwent a detailed medical history, physical exam including evaluation of the curvature (dorsal, ventral, lateral). Evaluation of the angle and direction of the curvature was done by measuring the angle in two dimensions from a snapshot picture of the erect penis. None of the patients was treated with ill in the past. The counseling technique regarding treatment options for PD were the same throughout the study period, except for the addition of CCH ILI counseling in 2016 to the provided information. Patients were excluded from the injection program according to the guided recommendations provided by the International Consultation on Sexual Medicine (ICSM), namely that this treatment should be reserved to men with a stable curvature and with a curvature angle greater than $30^{\circ}$ and less than $90^{\circ}$, without hourglass deformity, calcified plaque or any proximally located plaque at the base of the penis [5]. Data regarding curvature angle, plaque size and location, sexual function, patient preferences for type of therapeutic intervention, and previously prescribed and provided treatments were extracted retrospectively retrieved. For ILICCH, XIAFLEX® was used for four treatment cycles, each cycle consisting of two injections and penile modeling by the patient. Surgical treatment was tailored according to the individual patient's PD characteristics (plication, graft, penile prosthesis, etc.). Quantitative data were summarized by the percentage of patients who were interested in receiving treatment, percentage of patients who underwent surgery, and percentage of patients who underwent ILIC when the treatment was made available. A Chi-squared test was used to identify significant differences in proportions between group A and group B. A p-value less than an alpha probability of 0.05 was considered significant. SPSS software was used for all statistical analysis.

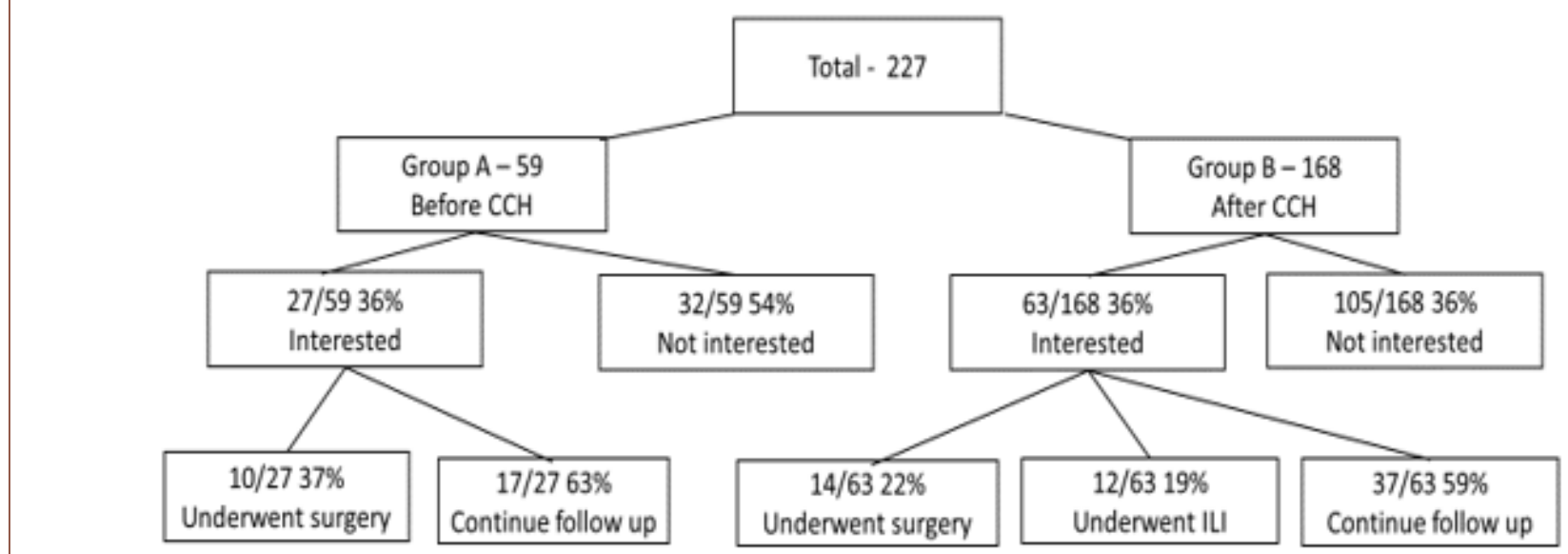

Interested = Interested to continue a follow-up program and treatment

Figure 1: Allocation of patients to groups. 
Two hundred and twenty-seven patients with stable PD were included in the study. Fifty-nine patients who were evaluated for penile curvature before the introduction of ILIC (group A), and 168 patients were evaluated after the introduction of ILIC (group B). Mean ages at the initial consultation were 60.6 years in group $A$ and 57.6 years in group B. Both groups were similar regarding the demographic and background diseases. In group A, after the first discussion on management of PD with a urologist, 27 (45.8\%) patients decided to pursue treatment ("Interested") and 32 (54.2\%) patients decided neither to pursue treatment nor any followup program ("Not interested"). In group B, 63 (37.5\%) patients decided to pursue treatment, and 105 (62.5\%) patients decided to pursue neither treatment nor any follow-up program (Figure 1).

There was no significant difference in the proportion of patients undergoing any treatment between the two groups $(p$ $>0.05)$. The main indication for intervention in both groups $\mathrm{A}$ and $B$ was a curvature that led to difficulties in sexual intercourse and vaginal penetration. At presentation, patients "not interested" in treatment from both groups A and B had minimal curvature (less than 100 angle) that interfered neither with sexual activity nor with emotional distress. In group A, of the remaining 27 patients who continued follow-up and were candidates for some type of intervention ("Interested"), 10 (37.0\%) underwent surgery (Figure 1). In group $B$, of the 63 remaining patients who continued followup ("Interested"), 14 (22.2\%) underwent surgery, while 12 (19.0\%) underwent ILIC (Figure 1). There was no statistically significant difference in the proportion of patients who underwent surgery between the two groups ( $p>0.05)$.

Of the 63 patients in group B who continued follow-up and were candidates for some intervention if deemed necessary, 26 (41.2\%) were unsuitable for ILIC therapy due to the type of deformity and plaque characteristics: three presented with a complex severe deformation (hourglass and beads type), five presented with a severe $(\geq 450)$ lateral deviation, four had a ventral deviation, and nine had severe calcifications. In one patient, the plaque was not amenable to injection due to its location at the dorsal penile base, imbedded in pre-public fat pad. In addition, two patients with concomitant severe erectile dysfunction were interested in penile implant, and two others requested maximal straightening of the penis, and as such were unsuitable for ILIC ultimately choosing surgery for treatment. Initially, only six eligible group B patients were interested in ILI of CCH. However, with worsening PD during the follow-up period, and further explanation of the pros and cons of both surgery and ILI, six more patients chose ILIC as their preferred treatment. By the end of the study period, 12 (19\%) of the group B patients interested in any treatment underwent ILIC and 14 (22\%) underwent surgery. Despite worsening PD, 37 (59\%) of the patients initially interested in any treatment, selected followup only without treatment (Figure 1). None of the patients treated with ILIC underwent surgery. See Figure 1 for detailed description of patient allocation to each group.

\section{Discussion}

Penile curvature and pain during erection present a significant bothersome issue for both men with PD and their partner [6-8]. Several treatment options are offered to the patients while surgery is usually delayed until PD stabilizes, a process that in most patients reaches its definitive state after a period around 18 months from the initial presentation of PD [9]. Among the non-surgical options, the only FDA approved drug for injection directly into the deformity-causing penile plaque is a collagenase enzyme derived from clostridium histolyticum. This medication was approved in 2013 for the treatment of PD in men with dorsal or lateral penile curvature greater than $30^{\circ}$ with or without a noncalcified penile plaque [5]. Current medicine practice emphasizes the patient's preferences and sovereignty for treatment selection. Whenever several treatment options become available with different outcome profiles and side effects, proper consultation and candid discussion with the patient regarding each treatment advantages and side effects are of paramount importance. The National Institute for Health and Care Excellence (NICE) in England recommends a shared decision-making process that enables physicians and patients to explore together the pros and cons of each treatment, and to tailor the treatment to individual requirements [10].Patients should be given a detailed explanation on the procedure and the technique in unbiased and plain language to ensure that their treatment choice is based on solid knowledge and on facts, and not on misinterpretation of the presented information. In the American Urological Association (AUA) guidelines on Erectile Dysfunction (ED), it is stated that "The Panel believes that shared decisionmaking is the cornerstone of the treatment and management of ED, a model that relies on the concepts of autonomy and respect for persons in the clinical encounter. It is also a process in which the patient and the clinician together determine the best course of therapy based on a discussion of the risks, benefits and desired outcome." We strongly believe that these recommendations also apply to the treatment of PD [11,12]. We conducted the present study to delineate whether the introduction of ILIC ty impacted the treatment patterns of PD. We also looked at the percentage of patients that in fact undergo ILIC out of all the patients evaluated for penile curvature at a men's health clinic. Surprisingly, in this study we were able to demonstrate that the introduction of ILIC had no significant effect on the percentage of patients who eventually undergo surgery. It seems that ILIC did not replace surgical treatment for PD in any way, but instead found its place as a preferred option over continuous follow up. In other words, patients that in the past were reluctant to undergo any penile surgery and preferred to remain with a distorted penis now had the option to receive a new form of treatment that, for them, seemed reasonable and acceptable with a good chance of partly improving their curvature. Following the appropriate consultation to patients pursuing follow up and treatment for PD, and eliminating those that were not eligible for ILI, only 12 out of the 63 evaluated patients 
that remained on follow up (19\%) underwent ILI as their initial treatment. A similar percentage of patients underwent surgery $(22 \%, p>0.05)$.

No statistically significant difference was demonstrated between Group A and group B regarding those patients who did not pursue further treatment or follow up $(\mathrm{p}<0.05)$. However, the total number of patients referred to our center increased from 2013 to 2019. This may be, in part, due to the introduction of ILIC, of which community urologists would have wanted to offer directly to their patients but given less familiarity with the pros and cons and less experience with the injection technique or protocol, preferred to refer out. Though this study did not aim to explore the reasons for the patients' choice, during our investigation we were exposed to their deliberation and debate regarding choice of treatment, which did not necessarily parallel the urologists' deliberations. A patient's choice of treatment may take into account information that the urologist does not emphasize. For example, Heisen reported that the physician may emphasize the benefits of a treatment, while the patient emphasizes the side effects [12]. Another explanation to our results may be the difference between the information provided by the referring physician and that provided by a specialist who is more focused and devoted to the field of PD. Levels of education as well as personal preferences for better success rates and fewer future procedures, as compared to a less invasive approach could all be factors that may affect the patient's choice of treatment. It may be that due to the quality of information available through the media, patients comprehended the advantages and drawbacks of follow up VS treatment, and as such, led to the substantial percentage of patients not interested in any mode of invasive treatment. In Israel, the cost of ILIC is mostly covered by medical insurance and none of the patients declined ILIC due to cost of treatment.

The addition of ILIC did not alter the percentage of patients undergoing surgery but increased the number of patients that underwent active treatment rather than follow-up only. Perhaps the combination of multiple factors i.e. proper selection of patients, open discussion, clear presentation of benefits and side effects, management of expectations from treatment and shared decisionmaking led to our current state showing that $19 \%$ of patients actually undergo ILIC and a similar percentage (22.2\%) undergo surgery. Future studies should consider evaluating these factors and investigating their true impact on the treatment pattern. Several limitations should be noted. Due to the study setting of a single medical center, the findings may not be pertinent to other urological centers. Some of the patients were referred by other urologists and as such, a selection bias by the referring urologist.
It is possible that only a patient looking for treatment or second opinion were referred. of the referrals could potentially affect our results. We do not have follow up data regarding the patients who chose to discontinue treatment at our center. It is inevitable that along the road some of them underwent some form of intervention or treatment. Further research is required to delineate the precise causes for patient preference although the information provided was equal in all patients.

\section{Conflict of Interest}

No conflict of interest.

\section{Acknowledgment}

Angelie Singh MD, MPH, MSc is thanked for editorial and linguistic assistance.

\section{References}

1. Mulhall JP, Schiff J, Guhring P (2006) An analysis of the natural history of Peyronie's disease. J Urol 175: 2115-2118.

2. Nelson CJ, Mulhall JP (2013) Psychological impact of Peyronie's disease: a review. The Journal of Sexual Medicine 10: 653-660.

3. Veale D, Miles S, Bramley S, Muir G, Hodsoll J (2015) Am I normal? A systematic review and construction of nomograms for flaccid and erect penis length and circumference in up to 15,521 men. BJU Int 115: 978986.

4. Love C, Katz DJ, Chung E, Shoshany O (2017) Peyronie's disease - Watch out for the bend. Aust Fam Physician 46: 655-659.

5. Chung E, Ralph D, Kagioglu A, Garaffa G, Shamsodini A, et al. (2016) Evidence-Based Management Guidelines on Peyronie's Disease. J Sex Med 13(6): 905-923.

6. Davis SN, Ferrar S, Sadikaj G, Gerard M, Binik YM, et al. (2016) Female Partners of Men with Peyronie's Disease Have Impaired Sexual Function, Satisfaction, and Mood, While Degree of Sexual Interference Is Associated with Worse Outcomes. J Sex Med 13(7): 1095-1103.

7. Davis S, Ferrar S, Sadikaj G, Binik Y, Carrier S (2017) Shame, Catastrophizing, and Negative Partner Responses Are Associated with Lower Sexual and Relationship Satisfaction and More Negative Affect in Men With Peyronie's Disease. J Sex Marital Ther 43(3): 264-276.

8. Ralph D, Gonzalez-Cadavid N, Mirone V, Perovic S, Sohn M, et al. (2010) The management of Peyronie's disease: Evidence-based 2010 guidelines. J Sex Med 7(7): 2359- 2374.

9. Randhawa K, Shukla CJ (2019) Non-invasive treatment in the management of Peyronie's disease. Ther Adv Urol 11: 1-13.

10. https://www.nice.org.uk/about/what-we-do/our-programmes/niceguidance/nice-guidelines/shared-decision-making.

11. Burnett AL, Nehra A, Breau RH, Culkin DJ, Faraday MM, et al. (2018) Erectile Dysfunction: AUA Guideline. J Urol 200(3): 633-641.

12. Heisen M, Baeten SA, Verheggen BG, Stoelzel M, Hakimi Z, et al. (2016) Patient and Physician Preferences for Oral Pharmacotherapy for Overactive Bladder: Two Discrete Choice Experiments. Curr Med Res Opin 32(4): 787-796 